\title{
A feasible method for clinical delivery verification and dose reconstruction in tomotherapy
}

\author{
J. M. Kapatoes ${ }^{\mathrm{a})}$ and G. H. Olivera \\ University of Wisconsin-Madison, 1300 University Avenue, Room 1530, Madison, Wisconsin 53706 \\ and TomoTherapy, Inc., 2228 Evergreen Road, Middleton, Wisconsin 53562 \\ K. J. Ruchala \\ TomoTherapy, Inc., 2228 Evergreen Road, Middleton, Wisconsin 53562 \\ J. B. Smilowitz \\ University of Wisconsin-Madison, 1300 University Avenue, Room 1530, Madison, Wisconsin 53706 \\ P. J. Reckwerdt \\ TomoTherapy, Inc., 2228 Evergreen Road, Middleton, Wisconsin 53562 \\ T. R. Mackie \\ University of Wisconsin-Madison, 1300 University Avenue, Room 1530, Madison, Wisconsin 53706 \\ and TomoTherapy, Inc., 2228 Evergreen Road, Middleton, Wisconsin 53562
}

(Received 24 April 2000; accepted for publication 2 January 2001)

\begin{abstract}
Delivery verification is the process in which the energy fluence delivered during a treatment is verified. This verified energy fluence can be used in conjunction with an image in the treatment position to reconstruct the full three-dimensional dose deposited. A method for delivery verification that utilizes a measured database of detector signal is described in this work. This database is a function of two parameters, radiological path-length and detector-to-phantom distance, both of which are computed from a CT image taken at the time of delivery. Such a database was generated and used to perform delivery verification and dose reconstruction. Two experiments were conducted: a simulated prostate delivery on an inhomogeneous abdominal phantom, and a nasopharyngeal delivery on a dog cadaver. For both cases, it was found that the verified fluence and dose results using the database approach agreed very well with those using previously developed and proven techniques. Delivery verification with a measured database and CT image at the time of treatment is an accurate procedure for tomotherapy. The database eliminates the need for any patient-specific, pre- or post-treatment measurements. Moreover, such an approach creates an opportunity for accurate, real-time delivery verification and dose reconstruction given fast image reconstruction and dose computation tools. (C) 2001 American Association of Physicists in Medicine. [DOI: 10.1118/1.1352579]
\end{abstract}

Key words: delivery verification, tomotherapy, dose reconstruction

\section{INTRODUCTION}

In previous works, it was shown that the incident energy fluence from a tomotherapy treatment could be verified by multiplying the treatment exit-detector sinogram by a measured transfer matrix. ${ }^{1,2}$ This process is referred to as delivery verification (DV). The transfer matrix is measured by opening each leaf of the multileaf collimator (MLC) individually, and recording the resulting signal profile in the exit detector for each leaf: The signal profile for each leaf represents a column of the transfer matrix. The measurements are made with the patient or phantom in the treatment position. Since tomotherapy treatments are rotationally continuous, transfer matrices are measured for each projection (beam angle) into which the delivery was divided by the optimization process. ${ }^{3,4}$ The matrices are then inverted. ${ }^{5,6}$ Finally, the detector signal profiles resulting from the group of pulses belonging to each projection are multiplied by the corresponding inverse transfer matrix to yield the incident energy fluence delivered by each leaf in every pulse for the entire rotational delivery.
With the verified energy fluence from DV and a computed tomography image (CT) of the patient in the actual treatment position, the dose deposited in the patient can be computed. This process is referred to as dose reconstruction (DR). ${ }^{4,7-10}$ This approach to DV and DR can be instrumental in treatment quality assurance and in elucidating delivery errors caused by the accelerator, MLC, patient positioning, anatomy changes, or a combination of all of these errors. ${ }^{7}$ Such delivery errors may lead to dosimetric errors that, when quantified accurately, could be corrected in subsequent fraction(s). This entire process is becoming known as adaptive radiotherapy.

With on-board imaging and verification capabilities, helical tomotherapy units will be capable of adaptive radiotherapy. Helical tomotherapy is a radiotherapy technique that employs a helically rotating fan beam of intensity-modulated radiation to deposit dose. ${ }^{4,11,12}$ Due to the large number of possible beams, tomotherapy treatments are optimized ${ }^{3,4}$ and are highly conformal for well-defined targets. For ill-defined targets, treatments conformally avoid sensitive structures 
while depositing dose to a large regional field(s). ${ }^{13}$ Helical delivery is accomplished by mounting the accelerator on a ring gantry and rotating the gantry while the patient is continuously transported through the bore. Intensity modulation is achieved through a binary MLC. A CT-style detector is mounted on the gantry distal to the patient. This integration of equipment on a single gantry allows for a host of verification processes. Specifically, patient registration in projection space, ${ }^{14,15}$ delivery modification without the need for reoptimization, ${ }^{16}$ megavoltage CT imaging (MVCT), ${ }^{17-20}$ DV, ${ }^{1,2,7}$ and $\mathrm{DR}^{4,7-10}$ will be possible.

The transfer matrix approach to DV and DR described previously are verification techniques for general radiotherapy and dynamic intensity modulated radiation therapy as well as tomotherapy: In principle the processes and that described in this manuscript can be used for other delivery strategies (e.g., those using dynamic MLCs). In fact, these processes belong to a larger class of verification techniques developed for radiotherapy using detectors located in an exit plane behind the patient. ${ }^{21}$ These include techniques using film and/or diodes, ${ }^{22-30}$ scintillator-camera based electronic portal imaging devices (EPIDs), ${ }^{31-36}$ and liquid-filled ion chamber based EPIDs. ${ }^{37-42}$ In comparison with these approaches, there are two main advantages of the transfer matrix method. First, signal, not dose, is related to the energy fluence. Such an approach is advantageous as extensive knowledge regarding the detector's dose-response characteristics is not required, which can be complicated in a xenon detector (such as that used in this work), or any solid state or liquid-based detector. Second, there are no assumptions regarding scatter in the detector plane since the transfer matrix is measured. This implies that the primary and scatter characteristics at each beam angle for every leaf are contained implicitly in the matrices.

In spite of these positive features, clinical implementation of the transfer matrix approach is hampered by the following:

(1) the time required measuring the transfer matrix with the patient on the treatment table,

(2) the computational time required calculating the energy fluence using the pulse-by-pulse treatment detector sinogram,

(3) the memory and hard disk space required storing the pulse-by-pulse treatment detector sinogram.

In this paper, a method is proposed that remedies the abovementioned issues. The first issue is addressed by using a measured database of signal profiles to create the transfer matrix. Such a database would eliminate any patient-specific, pre- or post-treatment measurements to create the transfer matrices. This database is a function of two parameters: the radiological path-length (RP) and the distance from the patient to the detector (DPD). These values are obtained for each projection from a CT of the patient/phantom in the treatment position. ${ }^{17-20,43}$ The consequences of not having a CT that accurately represents the patient/phantom in treatment position are discussed elsewhere. ${ }^{44-46}$ It will be shown that this database approach to DV provides results that are nearly identical to those with a measured matrix, which in principle is the most accurate technique and is thus used as a benchmark.

The second and third issues outlined previously are dealt with by using the projection-by-projection cumulative signal for delivery verification, rather than the pulse-by-pulse signal. This reduces the number of computations and storage space by a factor proportional to the number of pulses delivered in each projection (typically on the order of 100). The main difference between the approaches involves the tongueand-groove/penumbra (TAG-P) correction. However, it will be shown that the projection-by-projection results are nearly identical to those computed from the pulse-by-pulse technique, allowing for accurate and fast DV.

\section{THEORY}

\section{A. Delivery verification and dose reconstruction}

The theory of this approach to delivery verification and dose reconstruction has been described by Kapatoes et al. ${ }^{1,7}$ but will be summarized here. For either one accelerator pulse or a full projection, the signal $s$ in any detector element $i$ can be represented as a weighted sum of the energy fluence $\psi$ emitted from each MLC leaf, the weight being $d_{i j}$ :

$$
s_{i}=\sum_{j} d_{i j} \psi_{j},
$$

where the sum is over all $J$ leaves: $j=[1,2, \ldots, J]$. This equation can be written to include all $I$ detector elements by expressing it in vector-matrix notation:

$$
\mathbf{s}=\mathbf{D} \psi \text {. }
$$

Here, $\mathbf{s}$ is the vector of signal, $\boldsymbol{\psi} \boldsymbol{s}$ is the vector of energy fluence, and $\mathbf{D}$ is the matrix of signal in the detector per unit of energy fluence from the MLC. Thus a linear model is used to relate the detector signal and the energy fluence. For example, a $20 \%$ increase in the incident energy fluence will lead to a $20 \%$ increase in the detector signal. The reader should note that a linear relationship between detector dose and energy fluence in not required. A full discussion of linear systems can be found in Barrett and Swindell. ${ }^{47}$ The energy fluence can be obtained by multiplying the inverse of this matrix by the signal:

$$
\boldsymbol{\psi}=\mathbf{D}^{-1} \mathbf{s} \text {. }
$$

With the incident energy fluence from Eq. (3) and a CT in the treatment position, the dose can be computed using any model-based algorithm. Convolution/superposition was used in this study.

An important point regarding these equations is the corrections that must be incorporated to use the linear model described by Eq. (3). Namely, corrections for tongue-andgroove/penumbra (TAG-P) and leakage and transmission must be made. If not corrected large fluence errors will result. $^{1}$ 


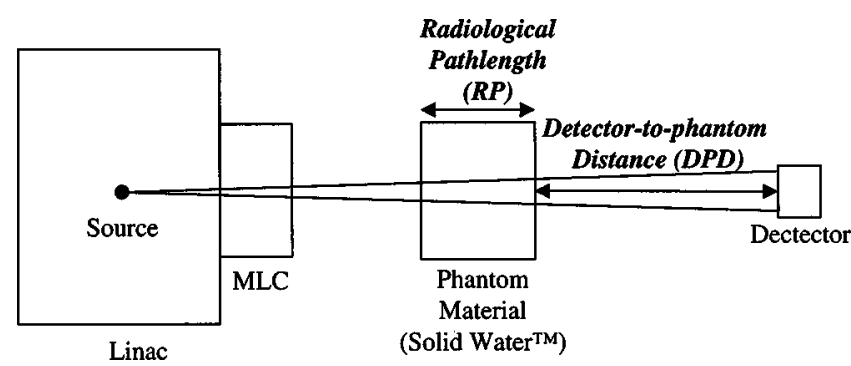

FIG. 1. Side view of the setup used to measure the database of signal profiles. The database was measured for 28 different radiological path-lengths (RPs) and three detector-to-phantom distances (DPDs). These parameters are computed from a $\mathrm{CT}$ at the time of treatment, eliminating the need to measure the profiles with the patient on the table. Different RPs were created by placing known amounts of Solid Water in the path of the beam. The leaves were cycled one by one with the beam on to collect signal profiles for every leaf at every combination of RP and DPD.

\section{B. Database}

The signal collected by a detector behind the patient is a function of the primary and scatter radiation received by the detector. Previous studies have shown that the exit signal from a radiotherapy beam can to first order be expressed as a function of two parameters: the radiological path-length (RP) and the distance from the patient to the detector (DPD). ${ }^{4-50}$ For this work, RP and DPD were computed from the CT at the time of treatment using a ray-tracing algorithm developed in-house based on Siddon's algorithm. ${ }^{51-53}$ For any given ray, the RP is defined by

$$
R P=\rho_{1} L_{1}+\rho_{1} L_{1}+\cdots+\rho_{n} L_{n},
$$

where $\rho_{n}$ is the physical density of the $n$th voxel encountered by the ray, and $L_{n}$ is the physical length of the ray inside the $n$th voxel. This approach ignores the location of inhomogeneities within the patient. The full field size is divided into a large number of rays in both the $x$ direction (perpendicular to the central axis of the beam and direction of translation) and the $z$ direction (perpendicular to the central axis of the beam and parallel to the direction of translation). The RP and DPD are calculated for each ray, and the appropriate value is assigned to each detector element. The computation is performed assuming a point source. The effect on the signal due to an extended source will be on the order of $2 \%$ for the fan beam used in this study (Tomotherapy Benchtop, see Sec. III A). For the Tomotherapy Prototype, a flattening filter will not be present, further reducing the importance of an extended source. For this situation, the extended source is confined to the size of the beam at the target. The effects of this extended source on the RP and DPD computation need not be considered: The ray paths from the extended source will fall within the resolution of the voxels of the CT volume since the source size is small (1-2 mm) and the voxels are relatively large $(3 \mathrm{~mm})$.

According to the exponential attenuation law, a radiation beam in narrow beam geometry attenuates exponentially according to the amount of material encountered between the source and detector. Thus, the RP will be an indicator of the amount of primary beam present at the detector. The scatter characteristics are analogous to those found in diagnostic imaging: The amount of scatter is reduced as the air gap (DPD) is increased. Based upon these physical principles, it was believed that a measured database of detector profiles could be used to accurately reconstruct the $\mathbf{D}$ matrix to relate signal to energy fluence in an energy-dependent detector such as xenon, allowing for DV and DR. The fact that the detector profiles are measured with the treatment unit implies that spectral dependencies are implicitly contained in each profile of the database.

\section{MATERIALS AND METHODS}

\section{A. Tomotherapy Benchtop}

All measurements were conducted on the Tomotherapy Benchtop. ${ }^{54,55}$ This consists of a $4 \mathrm{MV}$ General Electric (Buc, France) ORION accelerator, a NOMOS (Sewicky, PA) MiMiC MLC, a Parker-Hannifin (Rohnert Park, CA) automated actuator, and a General Electric (Milwaukee, WI) CT detector. The accelerator emits photons with an average energy of $1.37 \mathrm{MeV}$. For the MLC, only the top row of 20 leaves is utilized. The actuator is capable of simultaneous rotation and translation in two directions, simulating helical delivery. The CT detector contains xenon as the main filling gas. The detector is one dimensional and contains 738 channels, the central 500 of which are adequate to cover the MIMiC's field of view. Due to the small field size of the fan beam in tomotherapy, a one-dimensional detector can be considered sufficient.

A key feature of the Benchtop is that the accelerator and detector are not independent. Rather, the accelerator is pulsed and the detector read-off in a synchronized fashion. This allows for precise knowledge of where a pulse is delivered along the $360^{\circ}$ rotational path, which is necessary for the DV method of this work since a group of pulses are applied to one $\mathbf{D}$ matrix representing a projection (Sec. III B 3).

\section{B. Database}

\section{Measurement}

A database of detector signal profiles was measured by placing known amounts of Solid Water (RMI, Middleton, WI) perpendicular to the central axis of the beam, at various distances from the detector (Fig. 1). The Solid Water pieces were a combination of square $(15 \mathrm{~cm}$ by $15 \mathrm{~cm})$ and circular (30 $\mathrm{cm}$ diameter) slabs of varying thickness. The pieces were combined so that a total of 28 RPs were measured:

$$
\begin{aligned}
R P= & \{0.5,1.0,1.5,2.0,2.5,3.0,3.5,4.0,4.5,5.0,5.5, \\
& 6.0,7.0,8.0,9.0,10.0,11.0,12.0,14.0,16.0,18.0, \\
& 20.0,23.0,25.8,28.6,31.3,34.1,36.9\} \mathrm{cm} .
\end{aligned}
$$

This fine sampling of RPs was done since the detected signal was strongly dependent upon RP (exponential relation). The set of 28 RPs was measured for three detector-to-phantom distances (DPDs):

$$
D P D=\{12.0,22.0,35.5\} \mathrm{cm} .
$$


These DPD values are referenced to a plane perpendicular to the central axis at the point of the central detector element (though any plane beyond the furthest possible extent of the treatment volume would suffice). In this way the DPD values do not need to be computed for every detector element (even though the detector is curved). Once the Solid Water was in position, the leaves of the MLC would be cycled one by one. Enough open time was allowed for each leaf so that a large number of pulses could be collected (80), allowing for good statistics. Collecting the signal for each leaf completely filled the $\mathbf{D}$ matrix for that RP and DPD. Furthermore, by allowing time for accelerator warm-up (8-10 s) with the leaves closed, leakage and transmission information was also gathered for each RP and DPD (using pulses from $t=11.65 \mathrm{~s}$ $\rightarrow t=11.95 \mathrm{~s}$ : the cycling of the leaves began at $t=12.0 \mathrm{~s}$ ).

\section{Accessing the database}

Using a MVCT at the time of treatment, ${ }^{17-20}$ the RP and DPD values were computed for both the simulated prostate and canine nasopharyngeal deliveries (Sec. IIID). Because the $360^{\circ}$ delivery space is divided into a finite number of equiangular projections in the optimization process, ${ }^{3,4} \mathbf{D}$ matrices are formed for each of these projections (each projection contains a number of pulses or views). Thus, RP and DPD values were computed for every projection. These values were used to access the database and produce the projection-by-projection D matrices. For values that did not exactly equal a measured RP, log-linear interpolation was used:

$$
s_{\text {interp }}=\left(s_{1}\right)^{w_{1}} \times\left(s_{2}\right)^{w_{2}},
$$

where $s_{\text {interp }}$ is the interpolated signal value, $s_{1}$ is the signal value of the next-smaller measured RP from the database, and $s_{2}$ is the signal value of the next-greater measured RP from the database. $w_{1}$ and $w_{2}$ are the weighting value of $s_{1}$ and $s_{2}$, respectively, and are analogous to those used in linear interpolation. For example, the weights for RP interpolation are

$$
w_{1}=\frac{R P_{2}-R P_{\text {interp }}}{R P_{2}-R P_{1}}, \quad w_{2}=1-w_{1} .
$$

For DPD values, linear interpolation was utilized. For the cases in which both RP and DPD were between measured values, interpolation of RP was performed first, followed by that for DPD. It should be noted that with the level of RP sampling done for this database, the order of interpolations is less important than for a more sparse RP sampling.

\section{Projection considerations and database implementation}

Signal is acquired on a pulse-by-pulse basis (under continuous rotation), while $\mathbf{D}$ matrices are created on a projection basis (for a given angle). A typical treatment will have 100 pulses delivered in each projection. This inconsistency is remedied by multiplying the group of pulses in a particular projection by the single $\mathbf{D}$ matrix for that projection. This yields pulse-by-pulse energy fluence results. All fluence val- ues that correspond to a particular projection are then averaged together to provide the projection-by-projection fluence results. The number of pulses over which a leaf is opened is directly related to its planned energy fluence value. From an implementation perspective, for the arc around a given projection angle, each leaf is opened symmetrically around the given projection angle for an angle proportional to the planned fluence.

It has been previously shown ${ }^{7}$ that when $\mathbf{D}$ was measured with a number of pulses spanning the arc equal to one projection, it was necessary to weight the pulses used in creating D according to the planned energy fluence for each leaf. For example, given a delivery with 100 pulses per projection, consider a leaf that was to be opened for 60 pulses. For this case, the first and last 20 pulses would be given a weight of zero, and the 60 middle pulses would be given a weight of one. In this way, the pulses delivered over the section of the arc for which a leaf was to be opened are used to create that leaf's column in the $\mathbf{D}$ matrix. This technique provides more accurate results as the RP and DPD can change over the arc defining one projection. Such RP and DPD changes are most likely to occur in sections of the body that are sloping with regards to the external contour and/or interspersed with bone/tissue, air/tissue, or bone/air interfaces.

This approach can be simulated using the database. Instead of calculating one set of RPs and DPDs at the center of each projection, multiple sets are computed. Thus each projection is divided into sectors. The signal profiles for each sector are then weighted according to each leaf's optimal energy fluence value. There are two issues regarding the number of sectors created. First, the maximum number of sectors that would ever be needed is the number of pulses in one projection. However, creating this many sectors is not warranted due to the limitations imposed by the finite size of the voxels and would be a strain computationally. Second, only an odd number of sectors should be computed so that RP and DPD values for the center of the arc are maintained since leaf openings are centered on the arc and the optimization software computes dose for the center of each arc in creating an optimized plan. ${ }^{3,56}$ This approach is mathematically written as

$$
\mathbf{D}^{(p)}=\sum_{j=1}^{J} \sum_{k=1}^{N_{p}} w_{j}^{\left(p_{k}\right)} \mathbf{D}_{j}^{\left(p_{k}\right)},
$$

where the superscript $p$ denotes the projection number, $N_{p}$ is the total number of sectors in projection $p, w_{j}^{\left(p_{k}\right)}$ is the weight of the $k$ th sector for leaf $j$ in projection $p$, and $\mathbf{D}_{j}^{\left(p_{k}\right)}$ is the $j$ th column of the $\mathbf{D}$ matrix for the $k$ th sector in projection $p$. This method is illustrated for a single projection in Fig. 2. For this drawing, there have been three sets of RP and DPD values computed, thus the projection is divided into three sectors. Leaf A is to be opened for the entire projection (implying a large energy fluence prescription), thus the signal profiles calculated for each sector should be weighted equally. Leaf B will be opened for $75 \%$ of the projection. For this leaf, the relative weight of the profile for the sector 2 should be $(0.33 / 0.75=) 0.44$ since the leaf is opened during 


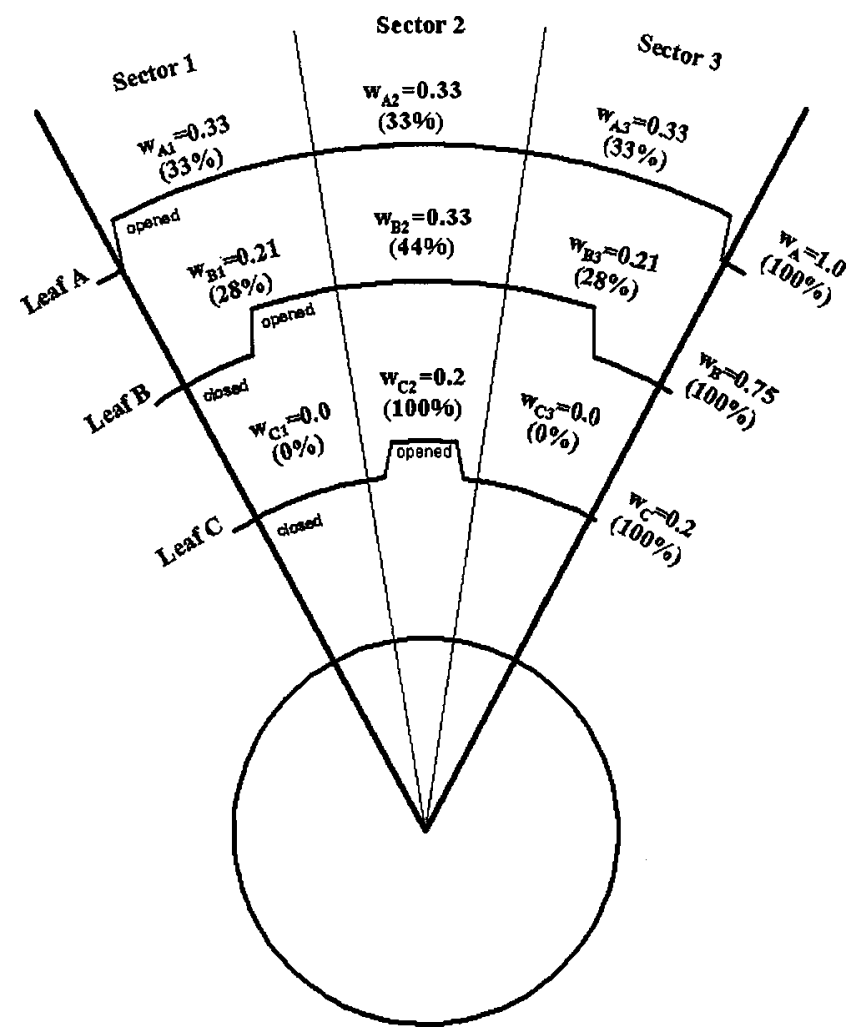

FIG. 2. Weighting scheme used for creation of the $\mathbf{D}$ matrix. Each treatment projection from the optimization process is divided into a number of sectors (shown here, three). Signal profiles are then extracted from the database for each sector, and these signal profiles are weighted based upon the how long a leaf is to be opened in that projection. Leaves A, B, and C are to be opened for $100 \%, 75 \%$, and $20 \%$ of the projection; the corresponding weights for the signal profiles in each sector are given in absolute and relative terms.

this entire sector. The remaining open time [which is equal to $(0.75-0.33)$ since 0.33 is expended in sector 2] is split evenly between the first and third sectors, therefore the relative weight is

$$
\left(\frac{(0.75-0.33) / 2}{0.75}=\right) 0.28
$$

for both sectors. Finally, leaf C is to be opened $20 \%$ of the projection or only in sector 2 . This implies the profiles for sector 2 should be given a relative weight of 1.0, and those from the first and third sectors should be given 0.0 weights. This can be extended for any arbitrary number of sectors per projection.

\section{Approaches to delivery verification}

\section{Pulse-by-pulse energy fluence}

Originally, we used Eq. (3) to obtain verified energy fluence values by applying the appropriate $\mathbf{D}$ matrix by the pulse-by-pulse signal. Results will be provided using this same approach with measured and database $\mathbf{D}$ matrices. The pulse-by-pulse approach is the most accurate because the tongue-and-groove/penumbra (TAG-P) correction is applied to the detector signal on a pulse-by-pulse basis. ${ }^{1}$ This is the most accurate method because the state of each leaf (opened, closed, or in transition) for every pulse is considered in deciding the appropriate correction.

\section{Projection-by-projection energy fluence}

A different approach than the pulse-by-pulse method can be pursued, which can reduce the required memory (both RAM and hard-drive storage) and thus increase the speed of the computations significantly. As there are typically 100 pulses per projection, the gain will be a factor of 100 for a typical treatment. The approach involves adding the signal over a projection, and multiplying the projection-byprojection $\mathbf{D}^{-1}$ matrices to the projection-by-projection signal. This will provide the projection-by-projection verified energy fluence. However, by doing this the TAG-P correction must be incorporated into the projection-by-projection $\mathbf{D}$ matrices, rather than the detector signal as in the pulse-bypulse approach.

The projection-by-projection TAG-P correction is performed for each leaf, over all projections. First, the cumulative signal profile for each leaf is divided spatially in half (where the central element of the profile is the center of the high signal region). Second, weighting factors are created for each half-profile based on the amount of time the nearest neighbor of each half-profile is opened. For example, consider a leaf that is to be opened for 50 pulses in a projection containing 100 pulses. Consider further that the nearest neighbor to the left is to be opened for 30 pulses and the nearest neighbor to the right for 80 pulses. The half-profile corresponding to the neighbor on the left will be TAG-P corrected for 30 of the 50 signal profiles of the cumulative signal, and the half-profile corresponding to the neighbor on the right for all 50 signal profiles of the cumulative signal. By TAG-P corrected, it is meant that the signal values for the detector elements affected by the presence of the tongue or groove of the leaves is increased to compensate for the loss of signal caused by the tongue or groove when a leaf is opened individually.

This projection-by-projection TAG-P correction is in principle less accurate than a pulse-by-pulse TAG-P correction for two reasons. First, transition states are not handled properly. Transition states are those pulses during which the leaf is in motion moving from a closed state to an opened state, or vice versa. In the pulse-by-pulse method, detector signal is collected during transition states since the time between pulses is shorter than the motion time. Therefore, approximate location of the leaf is known via these signal profiles, and the amount of signal increase for elements in the TAG-P areas can be scaled appropriately for leaves in transition. ${ }^{1}$ For the projection-by-projection approach, this information is not available and an approximate TAG-P correction must be applied to the $\mathbf{D}$ matrices based on the planned sinogram. However, since the transition in the opening direction tends to nearly compensate for that in the closing direction, and since transition states generally make up a small portion of the leaf states, the resultant errors were expected to be small. Second, by using the planned sinogram, 
the actual states of the leaves during delivery are not used and if there are significant leaf errors (e.g., a leaf is opened or closed too long), then the TAG-P correction based on the planned energy fluence values will be wrong.

\section{Projection-by-projection exit detector signal}

Rather than verifying the energy fluence during a treatment, it may be possible to verify the detector signal. In this approach, the projection-by-projection $\mathbf{D}$ matrices are used to compute what the projection-by-projection cumulative signal will be. This is accomplished by multiplying each leaf by the fraction of time it is opened in each projection (according to the planned sinogram), and then summing the leaf contributions together over projections. The D matrices utilized are the same from Sec. III C 2, thus the equivalent discussion applies involving the TAG-P correction. Once the projection-by-projection signal is computed, it is then compared to those collected during treatment. The advantage of this method is the computational simplicity and subsequent speed with which it can be performed. This is thus very amenable to real-time verification. However, the computational gains with respect to both memory and time compared to real-time energy fluence verification are not great. Moreover, the disadvantage of signal verification is that energy fluence is not computed as with the method of Secs. III C 1 and III C 2, thus dose reconstruction cannot be performed if this technique alone is used. However, if energy fluence verification was prohibitively time consuming, a two-stage process could be used in which signal verification would be performed in real time. If the signal values did not sufficiently agree, then a full energy fluence computation could be performed postdelivery.

\section{Case studies}

\section{Simulated prostate delivery}

A simulated prostate delivery was carried out on an abdominal phantom [Computerized Imaging Reference Systems (CIRS), Norfolk, VA]. This phantom possesses an exterior contour similar to an abdomen and is mainly comprised of water-equivalent material. However, at the center and along two concentric circles of different radii there are plugs of varying density. These plugs possess density values that mimic various tissues in the human anatomy (specifically: lung, breast, bone, liver, muscle, and adipose). A megavoltage CT image (MVCT) of the phantom in the delivery position is shown in Fig. 3(a). The phantom is identical in other slices.

Prostate, rectum, bladder, and femur contours were drawn based upon the MVCT image [Fig. 3(b)]. A simulated prostate delivery was then optimized using helical tomotherapy software. There were 51 projections per rotation in the optimization, or $7.06^{\circ} \pm 0.07^{\circ}$ per projection. The phantom was carefully positioned on the Workbench as inaccuracies with respect to the position in the planning $\mathrm{CT}$ could result in delivery errors. For example, an angular error in a real prostate patient could cause a misplacement of high contrast objects (such as the femoral heads) into projections with large

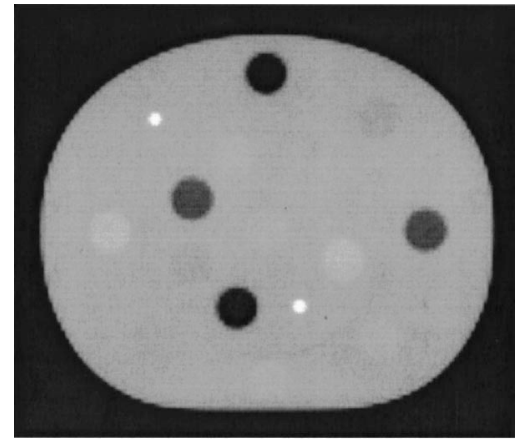

(a)

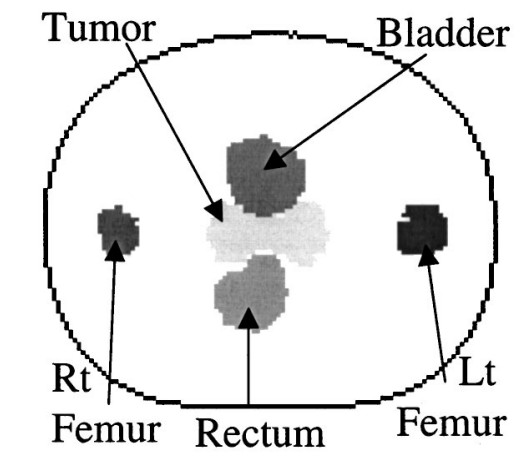

(b)

FIG. 3. (a) MVCT image of the CIRS abdominal phantom used for the simulated prostate delivery. (b) Regions of interest (ROIs) for the simulated prostate delivery drawn based upon the MVCT image in (a).

beam intensities, leading to possible fluence and dosimetric errors. Once set-up was complete, the phantom was helically treated over five slices with a helical pitch of 0.5 at a pulse rate of $200 \mathrm{~Hz}$. A pitch of 0.5 was used to minimize the dose junctioning artifacts present in tomotherapy. ${ }^{55,57,58} 500 \mathrm{~ms}$ were allotted to each projection, implying a rotation speed of $25.5 \mathrm{~s}$.

\section{Canine nasopharyngeal delivery}

In conjunction with the University of Wisconsin Veterinary School, a nasopharyngeal delivery was planned, delivered, and verified on a dog cadaver (German sheperd). The dose to the axis of rotation was approximately $38 \mathrm{cGy}$ as measured using a diode located in the nasal cavity. Helical CT data were acquired with a field size of $0.762 \mathrm{~cm}$ and slices were reconstructed that were $0.381 \mathrm{~cm}$ apart. MVCTs of two representative slices along with the corresponding regions of interest (ROIs) are provided in Fig. 4. The contoured tumor region spanned 32 slices (starting in slice 4), or a total distance of $12.2 \mathrm{~cm}$. The regions at risk included the nose, the left and right eyes, and the brain. The nose was present in slices $1-3(1.1 \mathrm{~cm})$. The right and left eyes (slices $25-30 ; 2.3 \mathrm{~cm}$ ) played a more influential role in the delivery as the tumor region lay between the eyes for these slices. Similarly, the brain (slices 29-39; $4.2 \mathrm{~cm}$ ) was in close proximity to the tumor. The optimization was carried out using 51 projections per rotation. Benchtop delivery parameters in- 

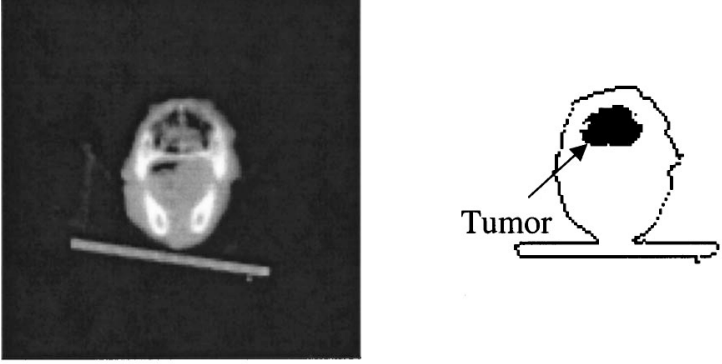

(a)

(b)
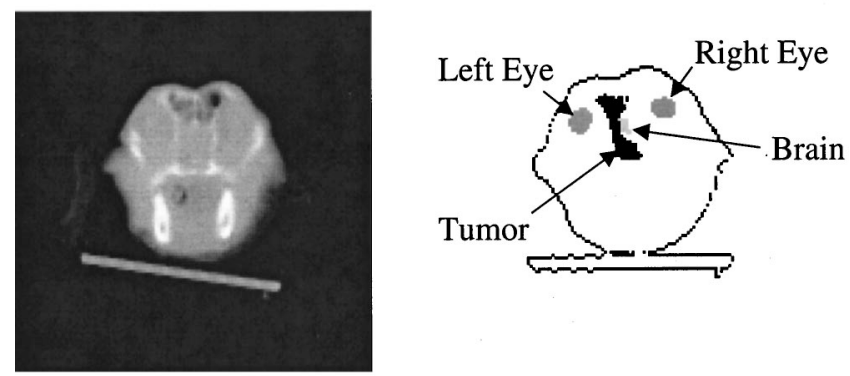

(c)

(d)

FIG. 4. MVCT images and ROIs for two representative slices of the canine cadaver head used in the nasopharyngeal delivery: (a) and (b) Slice 21. The tumor region is in a low-density area. This area is the sinus cavity of the dog. (c) and (d) Slice 29. The tumor region is surrounded by sensitive structures (both eyes and the brain).

cluded the following: helical delivery, $200 \mathrm{~Hz}$ pulse rate, 500 ms per projection, $25.5 \mathrm{~s}$ rotation speed, 0.5 pitch.

\section{RESULTS}

\section{A. Measurement and database}

\section{Matrix}

As an initial test of the database approach, a comparison of the measured and database $\mathbf{D}$ matrices is made in Fig. 5. Detector signal for the central detector element of two representative leaves from each delivery are shown. For the signal derived from the database, one sector (Sec. III B 3) per projection was used to construct $\mathbf{D}$.

A concern for any method that uses RP and DPD is that location of inhomogeneities is not considered. To investigate this effect, two parallel-opposed projections from a slice of the canine delivery were chosen that contained inhomogeneities close to the surface [Fig. 6(a)]. Database profiles for the two middle leaves were created for both directions. The boundaries of the leaves are provided in Fig. 6(a). In this slice, a bone is nearest the beam entry area for one projection, and for the parallel-opposed projection, a large air cavity is nearest the beam entry area. The database profiles were indistinguishable since the RP did not change (despite small DPD changes). One of these database profiles is plotted with the two measured profiles in Fig. 6(b). The profiles agree within $2 \%$ for the high signal detectors (channels 226-242).

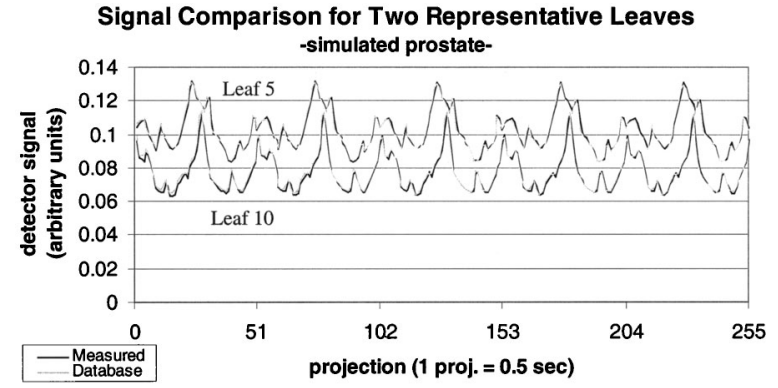

(a)

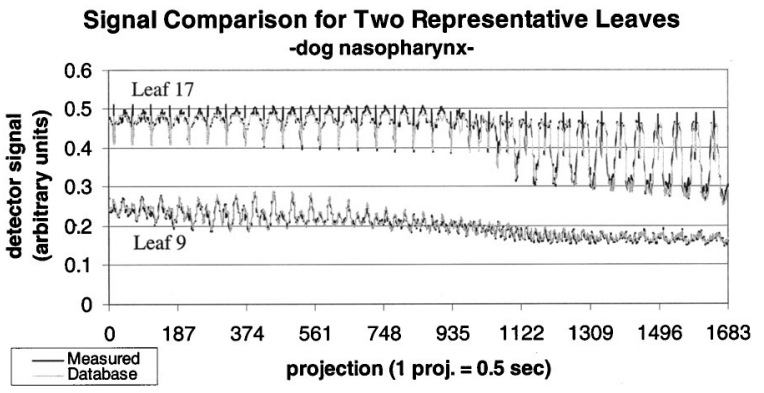

(b)

FIG. 5. Comparison for central detector element signal for two leaves. (a) Simulated prostate delivery. (b) Canine nasopharyngeal delivery. The "measured" plot was measured in the actual treatment position. The "database" plot was obtained from the database using the radiological pathlengths and detector-to-phantom distance data computed from the MVCT images (Figs. 3 and 4) taken at the time of treatment.

\section{Energy fluence}

Energy fluence verification was performed using the pulse-by-pulse approach for the measured and database D matrices. The measured verification sinogram, the database verification sinogram, and a difference sinogram are shown in Figs. 7 and 8 for the prostate and nasopharyngeal cases, respectively. For Figs. 7 and 8 the difference sinogram was produced by subtracting (b) from (a) and then dividing by the average energy fluence value for the active leaves of the measured sinogram (a). A histogram of these relative differ-

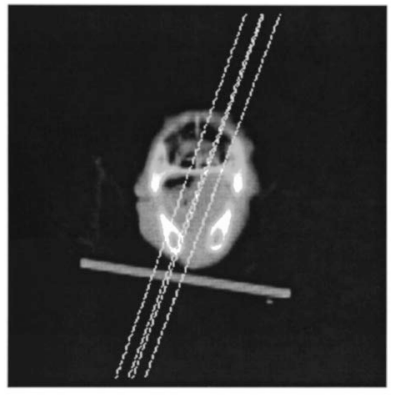

(a)

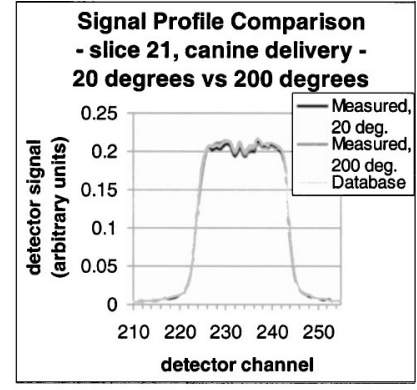

(b)
FIG. 6. (a) Slice 21 of the canine nasopharyngeal delivery in which detector signal from parallel-opposed projections was compared to examine the impact of the location of large inhomogeneities on the database method. The white lines detail the beam paths for the two middle leaves. (b) Measured signal profiles from the two projections compared with the database profile. The profiles are within $2 \%$ of each other, verifying that this issue does not affect the method to a large degree. 


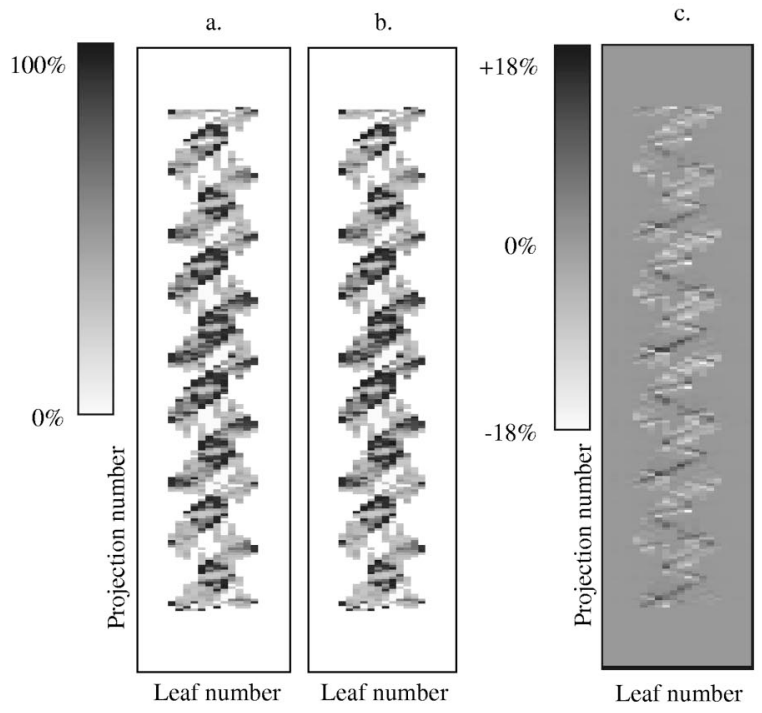

d.

Histogram of Energy Fluence Error -simulated prostate-

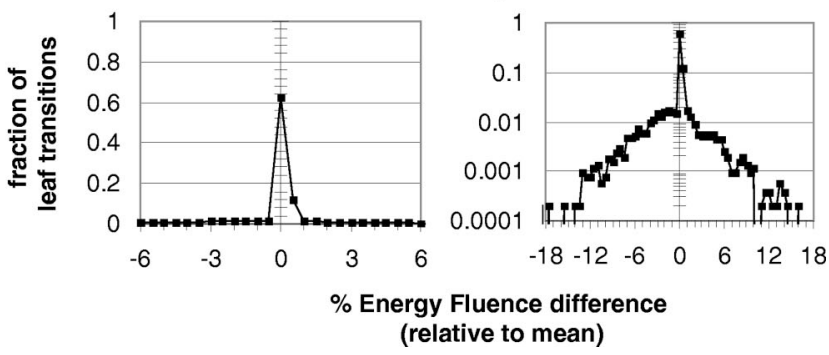

FIG. 7. Delivery verification (DV) results from the simulated prostate delivery. (a) Verification sinogram using the measured $\mathbf{D}$ matrix. In principle, measuring the matrix is the most accurate approach. (b) Verification sinogram using the database $\mathbf{D}$ matrix. (c) Difference sinogram, obtained by subtracting (b) from (a), and then dividing by the average nonzero planned energy fluence in (a). (a) Linear and log scale histograms of the difference values in (c). The differences are very small. A small fraction of leaves have larger errors underscoring the fact that the database approach will not give the same results as the "gold standard," measured-matrix technique.

ence values is shown with a linear scale and a logarithmic scale in (d). The linear plot is provided to illustrate that the overwhelming majority of errors are indeed small and the logarithmic plot is used to display the small fraction of leaves with larger errors.

Results were also generated using the database with three, five, seven, and nine sectors computed per projection, implying a $\mathbf{D}$ matrix every $2.35^{\circ}, 1.41^{\circ}, 1.01^{\circ}$, and $0.78^{\circ}$, respectively (Figs. 9 and 10). The difference sinogram for three sectors is provided in Fig. 9(a) for the prostate delivery. For Figs. 9 and 10 logarithmic histograms for all results are provided in (b). Linear histograms are not displayed since the differences between the approaches lay in the smaller fraction of leaves with larger errors, thus differences would be indistinguishable on such a linear scale.

\section{Detector signal}

Comparison of projection-by-projection cumulative signal is provided for the prostate and nasopharyngeal cases in
Figs. 11 and 12, respectively. For Figs. 11 and 12, the difference sinogram for one database-computed sector is provided in (a) and a logarithmic histogram summarizing all the results in (b). For both figures, the difference sinogram was created by subtracting the measured detector sinogram from the database detector sinogram, and then dividing by the average measured signal over the central detector elements for all active leaves. Again, linear histograms are not shown as the discrepancies are mainly in the small fraction of leaves with larger error. Such differences can only be distinguished on a logarithmic plot.

\section{B. Database delivery verification by pulse and projection}

Energy fluence verification using the pulse-by-pulse and projection-by-projection results are compared for the prostate and nasopharyngeal cases in Figs. 13 and 14 (scale has been changed with respect to the previous figures). Figures 13 and 14 possess the same format as Figs. 11 and 12: a difference sinogram for one sector comparing the two strategies is given in $a$ and a logarithmic histogram of the difference values for all results in (b). The difference sinogram was produced by subtracting the pulse-by-pulse verification sinogram from the projection-by-projection verification sinogram, and then dividing by the average energy fluence value for the pulse-by-pulse sinogram.

\section{Dose comparison}

Dose was computed using the verified energy fluence sinograms for some of the approaches. Specifically, dose was computed for the verification sinogram using the measured $\mathbf{D}$ [Figs. 7(a), 8(a)], the database $\mathbf{D}$ with one sector and the pulse-by-pulse approach [Figs. 7(b), 8(b)], the database D with five sectors and the pulse-by-pulse approach, and the database $\mathbf{D}$ with one sector and the projection-by-projection approach. CT images with the $30 \%, 50 \%$, and $90 \%$ isodose lines superimposed from the dose distributions are shown in Fig. 15. The simulated prostate results are displayed in row 1 [(a) -(c)], slice 21 of the canine nasopharyngeal case in row 2 [(d)-(f)], and slice 29 of the canine nasopharyngeal case in row $3[(\mathrm{~g})-(\mathrm{i})]$. For each figure, isodose lines using the measured $\mathbf{D}$ are given in white, and those from the distribution being compared are in black: The dose using the one-sector, pulse-by-pulse DV is compared in the first column [(a), (d), (g)], the dose using the five-sector, pulse-by-pulse DV in the second column [(b), (e), (h)], and the dose using the onesector, projection-by-projection DV in the third column [(c), (f), (i)]. Dose-volume histograms for all of these methods are shown in Fig. 16 for the prostate (a) and nasopharyngeal (b) deliveries.

\section{DISCUSSION}

The matrix comparison for the two representative leaves (Fig. 5) shows how well the database $\mathbf{D}$ matrix agrees with the measured $\mathbf{D}$ matrix. The agreement applies to both the overall pattern of the signal over time as well as the finer detail of the signal. The plots feature effectively how the signal reconstructed from the database follows very closely 


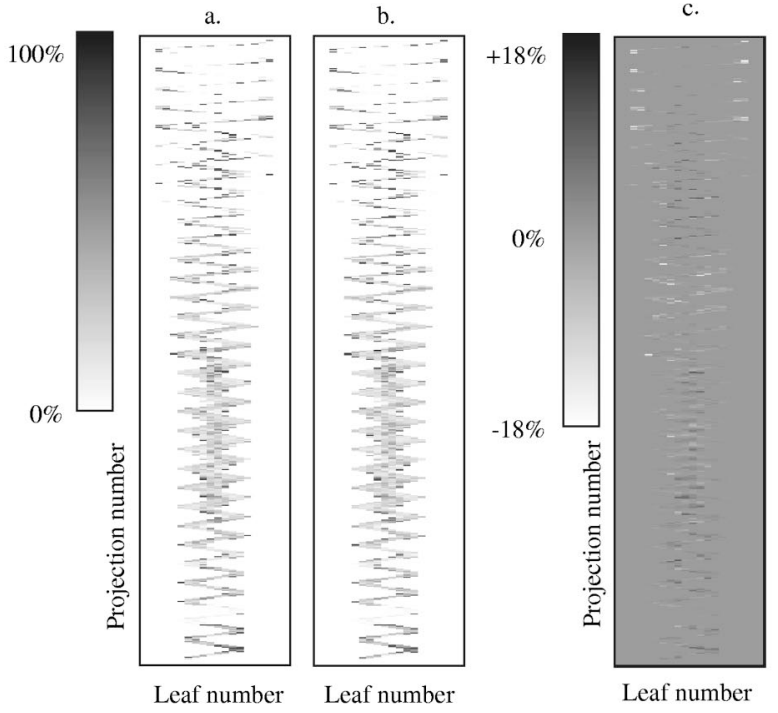

d.

Histogram of Energy Fluence Error -dog nasopharynx-

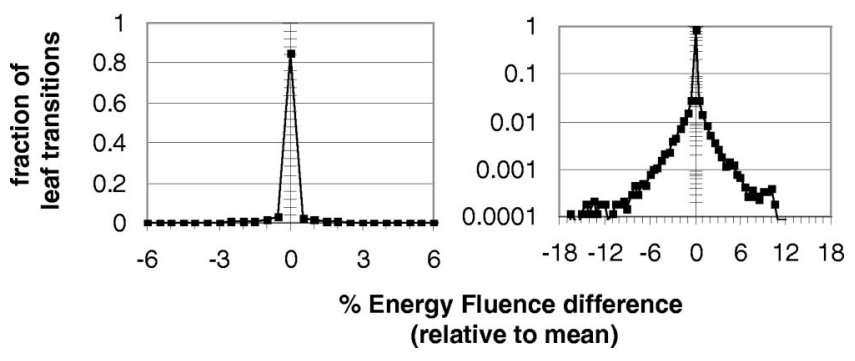

FIG. 8. Same as Fig. 7 for the canine nasopharyngeal delivery. Conclusions are unchanged.

the measured signal, even in places where there are abrupt changes in signal (implying abrupt changes in RP). Moreover, the agreement is worthy of mention due to the limited number of DPDs that were used for the studies (Sec. III B 1). This was done because it was found in a previous measurement in which many more DPDs were measured (step size was $3 \mathrm{~cm}$ ) that such a fine sampling of DPD was not needed. This is due to the small field sizes created by individual leaves, implying a small scatter-to-primary ratio and small variations in signal for changes in DPD.

The only area of noticeable disagreement in the signal comparison is the peaks of leaf 17 for the dog nasopharyngeal delivery [Fig. 5(b)]. This is a voxelization artifact of the database approach. This leaf's beam area happened to be on the edge of the dog's head, and thus there were moments during rotation in which the RP was equal to zero (signal value is just above 0.5 in the figure). However, for the database approach, the RP was never quite zero because voxels on the edge of the phantom had some density value due to partial volume effects. This is an inherent limitation of the method and may be problematic for database DV of treatments involving superficial targets.

The agreement between the measured and database profiles for the parallel-opposed projections in Fig. 6 is an example showing that the location of inhomogeneities does not affect the database method significantly. The fact that the profiles agreed to within $2 \%$ in spite of the beam direction, even though there was bone and an air cavity present, bodes well for the database approach. This result is most likely due to the small contribution of scatter for the fan beam in tomotherapy. A full study of this for various slice thickness and phantom geometries is under way. An extension to larger field sizes will also be attempted.

The fluence comparison was very important since this sinogram is needed for dose reconstruction. From the sinograms and histograms (Figs. 7 and 8) it is apparent that the differences in the verification sinograms are very small. In fact, $88.6 \%$ and $97.6 \%$ of the leaves for the prostate and nasopharyngeal deliveries, respectively, were within $\pm 3 \%$ of the values computed using the measured matrix. Therefore, the database approach was successfully applied to create the D matrix and compute the verified energy fluence values. This resolves the first issue raised in Sec. I: The database combined with the information from the $\mathrm{CT}$ obviate any patient-specific, pre- or post-treatment measurements needed to form $\mathbf{D}$, implying no added table time or extra patient dose.

A further issue regarding Figs. 7 and 8 is the smaller number of opened leaves per projection in the canine delivery relative to the simulated prostate. This was due to three issues with the canine nasopharyngeal delivery: the greater complexity of the case, the nonuniformity of the target volume in the inferior-superior direction, and the smaller size of the target in general. Moreover for the simulated prostate delivery, the middle leaves had a view of the target that was not impeded by sensitive structures for many projections. Even more interesting is the fact that the errors were not significantly larger for the prostate delivery in spite of the larger number of opened leaves per projection: The extra focal radiation is greater with more leaves opened simulta-
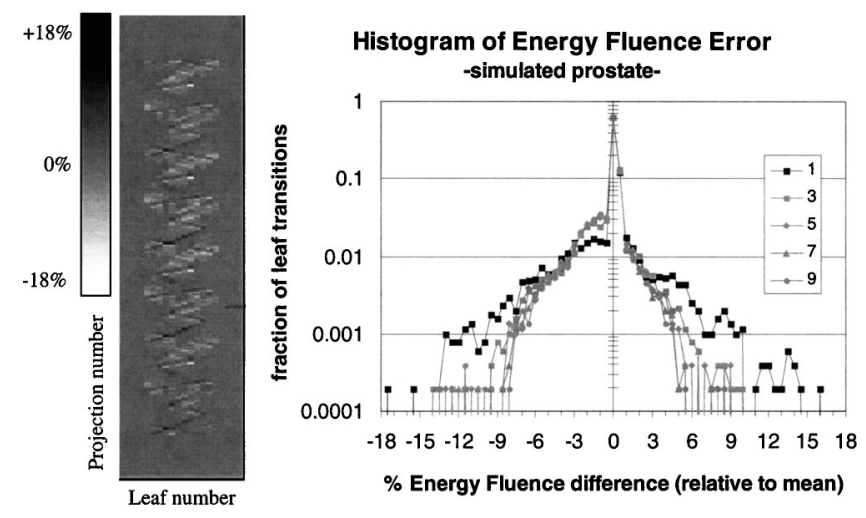

(a)

(b)

FIG. 9. Comparison of energy fluence DV results from the simulated prostate delivery for multiple sectors. The comparison is between results using the measured D matrix and the database-derived D matrix. (a) Difference sinogram for three sectors, showing noticeable improvement over the onesector approach [Figure 7(c)] (b) Histogram of difference values for varying sector number. The differences values between the approaches are small, and there is hardly any benefit (errors are not reduced) when using more than three sectors. Difference sinograms for more sectors are not shown since they were nearly indistinguishable from (a). 


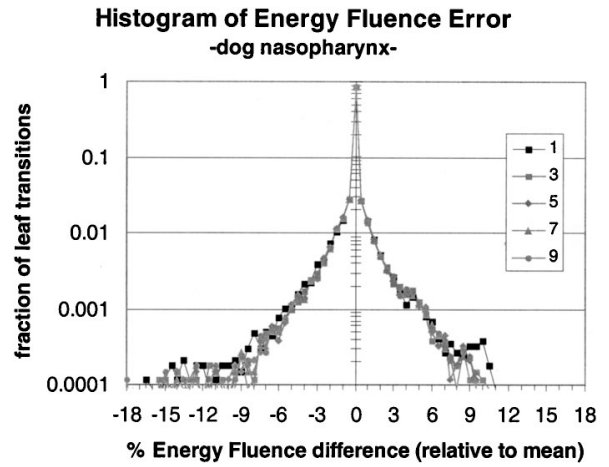

FIG. 10. Same as Fig. 9 for the canine nasopharyngeal delivery. The difference values between the approaches are even smaller than those for the simulated prostate delivery, and there is little benefit (errors are not reduced) when using multiple sectors. Difference sinograms for multiple sectors are not shown since they were nearly indistinguishable from Fig. 8(c).

neously. Because the database is measured one leaf at a time, one might expect that such a situation would lead to larger error. This was not the case.

Investigations into the possibility of dividing projections into a number of sectors proved interesting (Figs. 9 and 10). For the prostate delivery (Fig. 9), there is a clear benefit in using the multisector approach of Sec. III B 3: the difference values are visibly reduced in going from one sector [Fig. 7(c)] to three [Fig. 9(a)]. The benefit becomes more difficult to ascertain as the number of sectors increases as confirmed in the histogram of the difference values [Fig. 9(b)]: The curves for three to nine sectors overlap but all of these are improvements over the one sector curve. Since RP and DPD values are computed using the CT image, there is a fundamental limit to the improvement that can come from additional sectors. This limit is related to the size of the individual voxels in the CT image. Thus it should not be surprising to see the improvement taper off as the number of sectors are increased. However, increasing the number of (a)

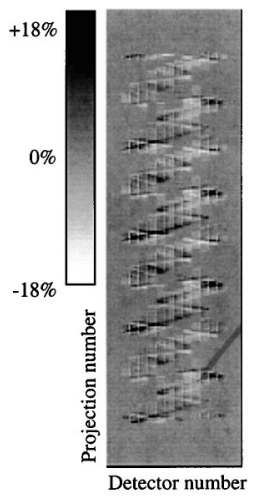

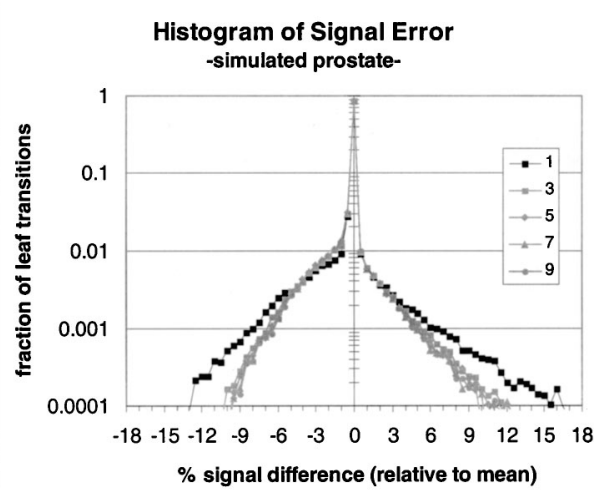

(b)
FIG. 11. Comparison of detector signal DV results from the simulated prostate delivery for multiple sectors. (a) Difference sinogram for one sector. The improvement in this sinogram when using three sectors is similar to that seen in Fig. 9(a). Larger errors are present (vertical streaks in particular) due to the differences in the high signal gradient regions between leaves. (b) Histogram of difference values for varying sector number, following the trend seen for energy fluence verification in Fig. 9(b).

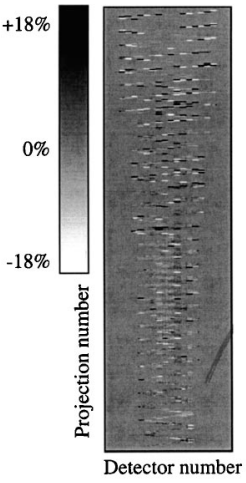

(a)

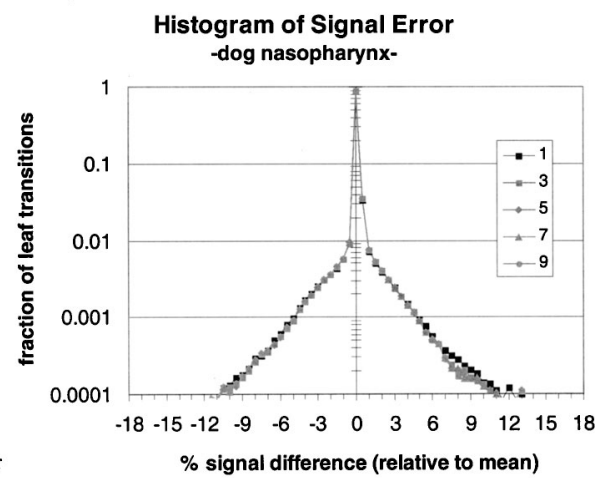

(b)
FIG. 12. Same as Fig. 11 for the canine nasopharyngeal delivery.

sectors did slightly improve the results. Specifically, the percentage of leaves with errors between $\pm 3 \%$ were $88.6 \%$, $94.0 \%, 94.8 \%, 95.1 \%$, and $95.3 \%$ for one, three, five, seven, and nine sectors, respectively. Thus, it appears from this case that there are definite gains to be made by increasing the number of sectors in computing $\mathbf{D}$, however there are diminishing improvements in using more than three sectors. For an actual prostate patient in which femoral heads will be present, the error levels should remain similar to those reported here for two reasons: (1) With enough sectors per projection, the effects of the femoral heads should be accurately characterized relatively well. (2) The optimizer will typically not use many beams that pass through the femoral heads since they are sensitive structures. Thus, although the fluence errors for such beams may be large relative to the prescribed value, the fluence error relative to the average fluence and the effects on the dose will be very small.

For the canine delivery (Fig. 10), the advantage of multiple sectors is less apparent. The difference sinograms for increasing sector number showed no visible variations from

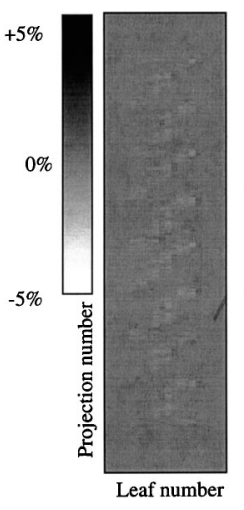

(a) (b)

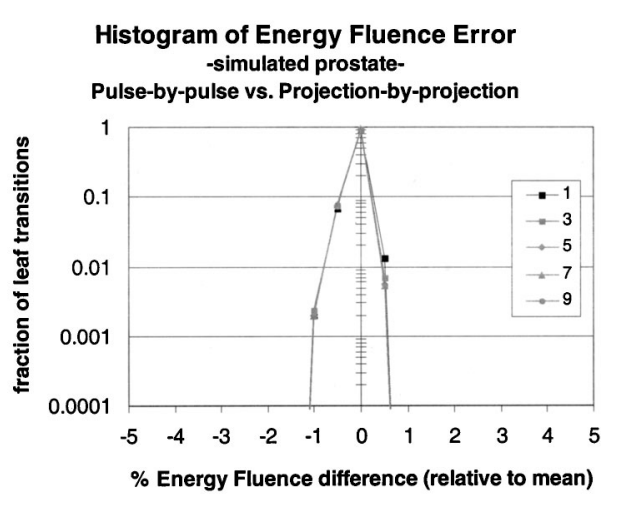

FIG. 13. Simulated prostate energy fluence DV results using the pulse-bypulse technique are compared with those using the projection-by-projection technique for varying sector number. (a) Difference sinogram for one sector. Difference sinograms for more sectors are not shown since they were nearly indistinguishable from (a). (b) Histogram of difference values for varying sector number. In principle, the pulse-by-pulse technique is more accurate. There appears to be very little difference between the results using the two techniques. 

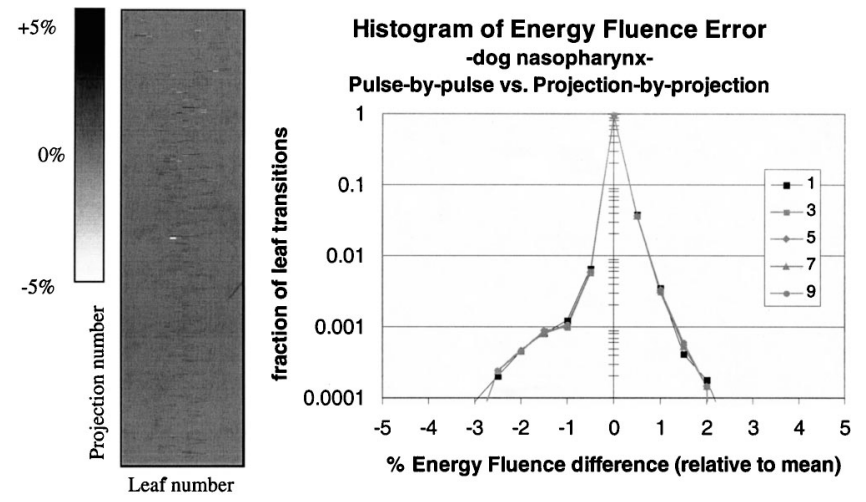

(a)

(b)

FIG. 14. Same as Fig. 13 for the canine nasopharyngeal delivery. Again, there is very little difference between the pulse-by-pulse and projection-byprojection techniques.

the one-sector sinogram provided in Fig. 8(c). This is substantiated in Fig. 10 as all curves are quite similar. It might intuitively seem that the conclusions from these cases are backward: A phantom with inferior-superior inhomogeneities and anatomically complex structures such as the dog should benefit more from multiple sectors. The key aspect here is the gradients in RP of the two cases. The radiological path-length for the dog's head does not change significantly for small angles while it does for the CIRS phantom. Thus, the radiological path-lengths will change more from one projection to the next for the CIRS phantom. This is seen in Fig. 5. The signal detected for the CIRS phantom fluctuates more than that for the dog with the exception of the signal for leaf 17 from projection 1000 to the end of the plot. However, attention should be focused on the central leaves (leaf 10 for the prostate and leaf 9 for the nasopharyngeal) as these leaves are used heavily in the deliveries since the tumor in both cases is located at isocenter. A reasonable conclusion from these observations is that multiple sectors are useful for regions of the body whose RP change significantly for small angles (e.g., thorax, abdomen) but may not be for RPsymmetric regions (e.g., head and neck).

Regarding the detector signal comparison, the trend of agreement between the measured and database methods is apparent in Figs. 11 and 12. Once again, there is a clear benefit to multiple sectors for the prostate delivery on the CIRS phantom [Fig. 11(b)]. The percentage of leaves between $\pm 3 \%$ was $96.4 \%$ for the prostate with three sectors, and $97.6 \%$ for the nasopharyngeal with one sector. This is an important result since the database signal was reconstructed and the TAG-P correction made on a projection-byprojection basis. All previous results were computed using

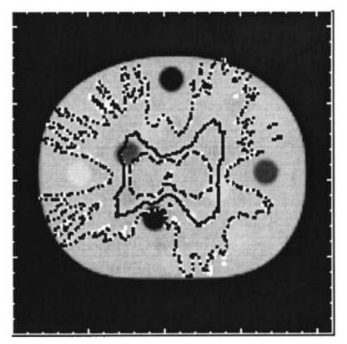

d

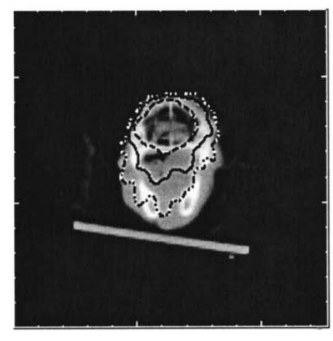

g

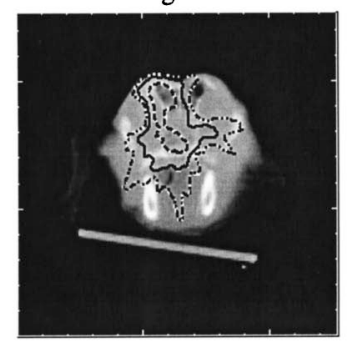

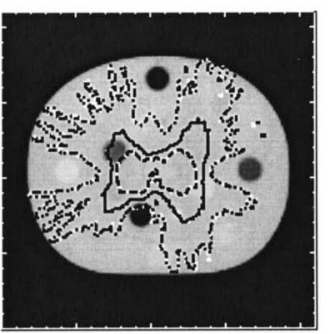

e
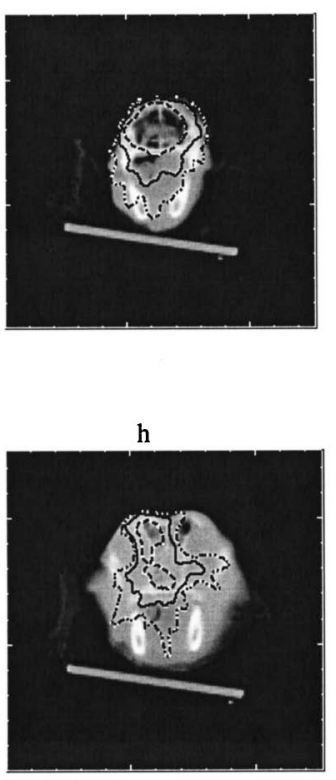

c

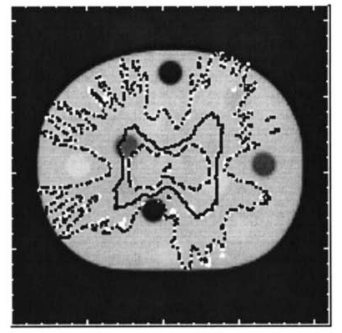

f
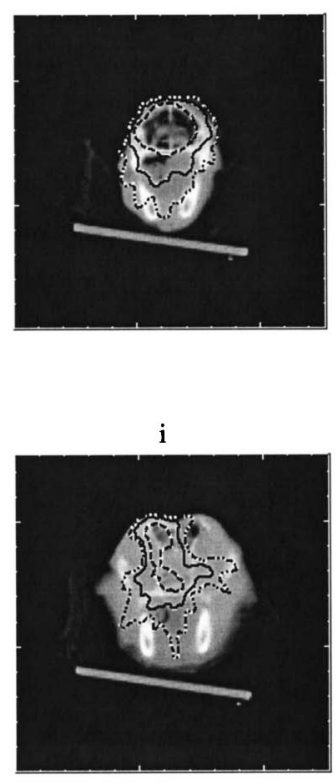

FIG. 15. CT images with $30 \%$ (dash-dot-dot), $50 \%$ (solid) and 90\% (dash) isodose lines superimposed for the simulated prostate (a)-(c), slice 21 of the canine nasopharyngeal (d)-(f), and slice 29 of the canine nasopharyngeal (g)-(i) deliveries. The isodose lines computed using the energy fluence from the measured $\mathbf{D}$ matrix are in white for all figures. The reconstructed dose using the energy fluence computed using different approaches with the database $\mathbf{D}$ matrix is in black for all figures. The pulse-by-pulse technique using one sector is compared in the first column $[(\mathrm{a}),(\mathrm{c}),(\mathrm{e})]$, the pulse-by-pulse technique using five sectors in the second column [(b), (d), (f)], and the projection-byprojection technique using one sector in the third column. 

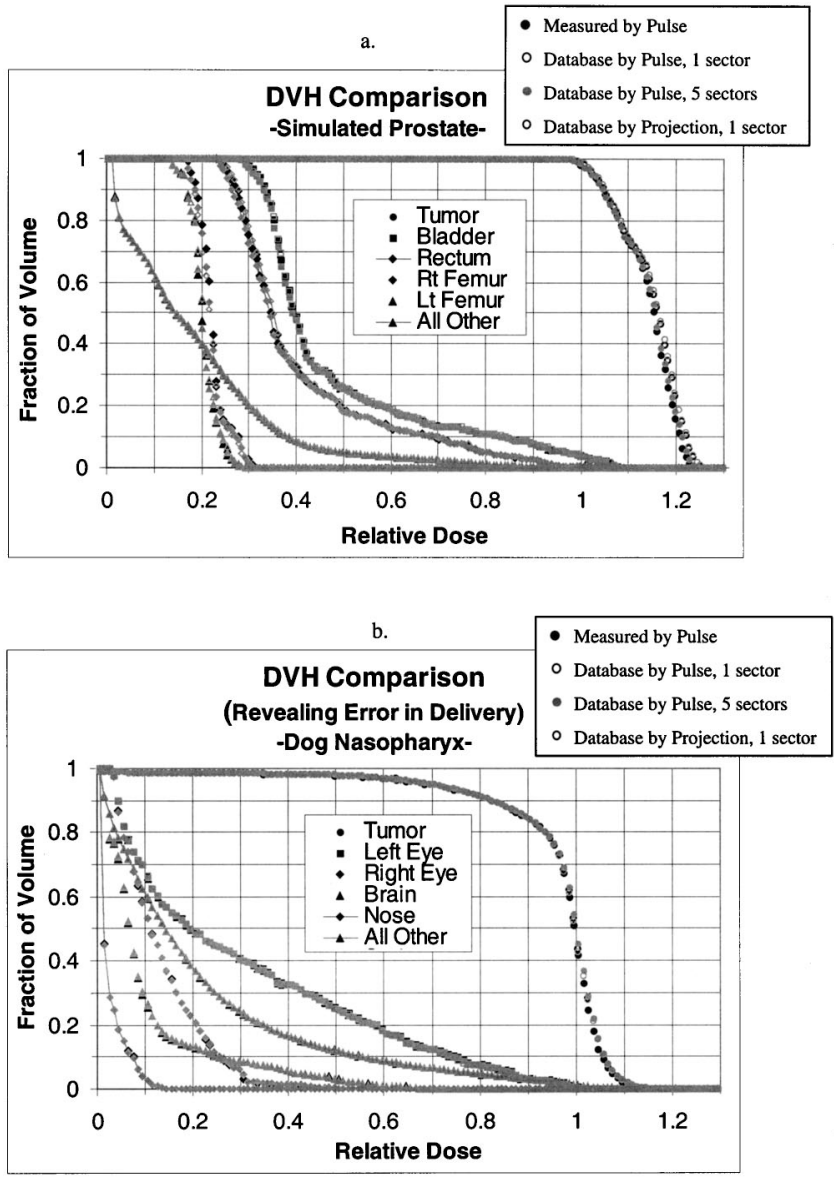

FIG. 16. Dose volume histograms comparison. (a) Simulated prostate delivery. (b) Canine nasopharyngeal delivery. The differences between the DVH lines for the dose distributions computed for the varying DV techniques are minor. The conclusions regarding the overall treatment would be the same regardless of which DV technique is used. This confirms that methods for real-time energy fluence verification, i.e., projection-by-projection energy fluence verification, can be used to perform accurate dose reconstruction.

the pulse-by-pulse technique. The fact that there is widespread agreement verifies that signal verification and TAG-P corrections can be accurately done projection by projection as well as pulse by pulse.

The final issue for delivery verification was projection-byprojection energy fluence verification. The agreement between the projection-by-projection and pulse-by-pulse strategies is clear in Figs. 13 and 14. It is important to recognize the scale change in Figs. 13 and 14 with respect to the previous figures: The error was very small so the scale was altered to $-5 \%$ to $+5 \%$. In fact, for the prostate and nasopharyngeal cases, all values were within $\pm 1 \%$ and $\pm 3 \%$, respectively. This implies that comparing the measured sinogram with the verification sinogram computed using the projection-by-projection strategy would yield results that almost match those using the pulse-by-pulse technique seen in Figs. 7 and 8. This is particularly interesting when one considers the projection-by-projection signal verification results, which showed some areas of large error [in particular, the vertical streaks in Figs. 11(a) and 12(a)]. These errors in signal verification arise from discrepancies in high signal gradient regions at the interfaces between leaves. With energy fluence verification, applying $\mathbf{D}^{-1}$ acts as a filter through which signals corresponding only to the particular leaf are passed and compared. Thus regions of high signal gradient are effectively filtered out allowing for focus to be placed on the main signal channels associated with each leaf.

The success of the projection-by-projection results allows for resolution of the second and third issues raised in Sec. I: computational time and memory demands of the process. Since there are typically 100 pulses per projection for a treatment, the computational time and memory requirements are subsequently reduced by a factor of 100 when the projectionby-projection approach is used, with insignificant losses in accuracy. As an example, for the dog delivery that included 40 slices and 168300 pulses, this gain turned a process requiring over 3 min and 450 Mbytes RAM, into a 2 s process requiring 4.5 Mbytes RAM.

The dose comparisons in Figs. 15 and 16 illustrate how indistinguishable the techniques are from a dosimetric standpoint. For the isodose comparison (Fig. 15), the isodose lines from the dose distributions computed using the different approaches (black) are positioned on top of the isodose lines using the measured D matrix (white). Furthermore, the DVH lines are so similar (Fig. 16) that the conclusions regarding the outcome of the treatment would be unchanged regardless of the technique used for DV. Since the accuracy of the dose reconstruction technique was previously shown for the pulseby-pulse measured matrix approach, ${ }^{7-9}$ Figs. 15 and 16 are dosimetric proof that a database approach to DV can be used to accurately compute the energy fluence necessary for dose calculation. Moreover, these figures show that a projectionby-projection strategy can be successfully applied, alleviating the computation time and memory burdens of a pulseby-pulse approach.

Figures 15 and 16 also illustrate further interesting conclusions regarding rotational delivery in general. It appears that the small discrepancies in energy fluence when using the measured $\mathbf{D}$ matrix versus the various database $\mathbf{D}$ techniques (seen in the previous figures) become "washed out" in the dose computation. That is, small errors in energy fluence tend to be compensated in the overall delivery by the large number of beams used from many directions. This is a positive result since MLC leaves are not perfect, and accelerators occasionally decrease output for a few pulses. Both phenomena will lead to small errors in the verified energy fluence that-over the course of $360^{\circ}$ rotational therapy-will not have a large impact on the overall outcome.

One issue that could have an impact on this approach to DV and DR is intrafraction motion. Such motion exemplified by the breathing motion could cause rapid and periodic changes in RP. Therefore, this would be a concern in thoracic and abdominal cases. For such cases, there are options. First, the treatment could be gated so that pulses were only delivered in positions that closely correspond to the CT taken at treatment time. A second option is to have fast-CT capabilities that would allow for images to be acquired for every projection. A final option is implement correction factors in the computed RP values based upon previous knowledge of 
the motion. This could be achieved by acquiring two images: one at expiration and one at full inspiration. The breathing could then be tracked during treatment and appropriate RP correction factors applied.

Finally, though unrelated to the core topic of this paper, a final comment regarding the dog nasopharyngeal case will be offered. The reader will notice that this DVH [Fig. 16(b)] is poor for two reasons: a significant fraction of the tumor is severely underdosed, and the left eye (relative to the right eye) is overdosed. This was not present in the DVH for the optimized dose (not shown) and therefore this was not an optimization problem. The error was caused by a rotational offset present in the delivery that is apparent upon comparison of the MVCT images [Figs. 4(a) and 4(c)] and the ROI images [Figs. 4(b) and 4(d)]. The magnitude of the offset was $8.7^{\circ}$ and was caused by a lack of synchronization between the actuator and the rest of the delivery system: the actuator started rotating 1-2 s late. The offset was discovered upon reconstruction of the MVCT image. There was an absolute position at which the image reconstruction began. Once the image was created, the rotation present in the treatment-time MVCT image with respect to the planning $\mathrm{CT}$ was noticeable. The magnitude of the offset was verified independently using the knowledge of the rotation speed and the delay period, and also by image fusion.

\section{CONCLUSION}

A method for delivery verification is presented that eliminates the need for patient-specific pre-or post-treatment characterization measurements with the patient on the treatment table and significantly reduces computational demands. The method, as implemented in a clinical tomotherapy treatment, is summarized in Fig. 17. A transfer matrix that allows for the transition from detector signal to incident energy fluence is computed using a measured database of signal profiles. An important feature of this approach is that signal-not dose-is related to energy fluence. This is in part possible because a CT at the time of treatment is available in tomotherapy, which in turn provides knowledge of the primary and scatter characteristics for every projection. This information is sometimes lacking in traditional verification techniques using portal imaging devices. The database is a function of the radiological path-length and detector-to-patient distance, both of which are computed from this CT image. One transfer matrix per projection is shown to be adequate for some cases, although it is illustrated that accuracy is increased when each projection is divided into multiple sectors for anatomies in which the radiological path-length changes rapidly as a function of angle. The pulse-by-pulse energy fluence computed using the database matrices is shown to be very similar to that using measured transfer matrices. Additionally, the dosimetric results using as input the energy fluence from the different approaches are the same.

To decrease the computational requirements, projectionby-projection delivery verification is presented. Such an approach would reduce the computational demands by a factor equal to the number of pulses in each projection (typically

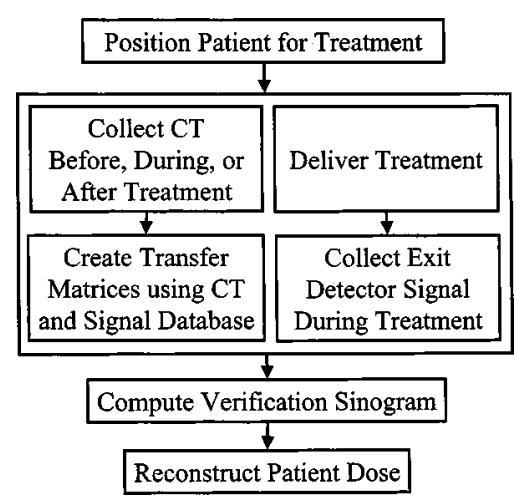

FIG. 17. Block diagram summarizing the steps involved in verifying the delivery and reconstructing the dose for a clinical tomotherapy treatment.

100). The projection-by-projection verification sinograms are shown to be nearly indistinguishable from those generated using the pulse-by-pulse technique. Again, the dose computations using either approach are almost identical. With computational burden significantly reduced at little expense to accuracy, real-time delivery verification is a definite possibility as is real-time dose reconstruction with a fast and accurate dose calculation.

\section{ACKNOWLEDGMENTS}

The authors would like to thank Lisa Forrest, D.V.M., John Balog, Ph.D., Harry Keller, Ph.D., and Dave Pearson, Ph.D., for their time and efforts with the dog delivery. J.M.K. would like to thank Eric Schloesser and Dave Pearson for their assistance with and upkeep of the Tomotherapy Benchtop. J.M.K. and T.R.M. are grateful for the support of the NCI-sponsored University of Wisconsin Radiological Sciences Grant No. T32-CA09206 and NIH Grant No. CA48902.

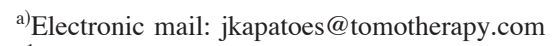

${ }^{1}$ J. M. Kapatoes, G. H. Olivera, P. J. Reckwerdt, E. E. Fitchard, E. A. Schloesser, and T. R. Mackie, "Delivery verification in sequential and helical tomotherapy," Phys. Med. Biol. 44, 1815-1841 (1999).

${ }^{2}$ J. M. Kapatoes, G. H. Olivera, P. J. Reckwerdt, E. A. Schloesser, E. E. Fitchard, D. W. Pearson, J. P. Balog, and T. R. Mackie, "Delivery verification in tomotherapy," Med. Phys. 25, A110 (1998).

${ }^{3}$ D. M. Shepard, G. H. Olivera, P. Reckwerdt, and T. R. Mackie, "Iterative approaches to dose optimization in tomotherapy," Phys. Med. Biol. 45, 69-90 (2000).

${ }^{4}$ G. H. Olivera et al., "Tomotherapy," in Modern Technology of Radiation Oncology, edited by J. Van Dyk (Medical Physics Publishing, Madison, 1999), pp. 521-587.

${ }^{5}$ H. Press, B. P. Flannery, S. A. Teukolski, and W. T. Vettering, Numerical Recipes in C: The Art of Scientific Computing (Cambridge University Press, Cambridge, 1988).

${ }^{6}$ J. H. Wilkinson and C. Reinsch, in Handbook for Automatic Computation, edited by F. Bauer (Springer, New York, 1971), Vol. 2.

${ }^{7}$ J. M. Kapatoes, G. H. Olivera, J. P. Balog, H. Keller, P. J. Reckwerdt, and P. J. Mackie, "On the accuracy and effectiveness of dose reconstruction for tomotherapy," Phys. Med. Biol. (in press).

${ }^{8}$ J. M. Kapatoes, G. H. Olivera, H. Keller, P. J. Reckwerdt, E. E. Fitchard, K. J. Ruchala, and T. R. Mackie, "Dose reconstruction in tomotherapy," Med. Phys. 26, 1104 (1999).

${ }^{9}$ J. M. Kapatoes, “www.madrad.radiology.wisc.edu/tomo/dose_recon/ dose_ren.html", 
${ }^{10}$ T. R. McNutt, T. R. Mackie, and B. R. Paliwal, "Analysis and convergence of the iterative convolution/superposition dose reconstruction technique for multiple beams and tomotherapy," Med. Phys. 24, 1465-1476 (1997).

${ }^{11}$ T. R. Mackie, T. Holmes, S. Swerdloff, P. Reckwerdt, J. O. Deasy, J. Yang, B. Paliwal, and T. Kinsella, "Tomotherapy: A new concept for the delivery of dynamic conformal radiotherapy," Med. Phys. 20, 17091719 (1993).

${ }^{12}$ T. R. Mackie et al., “Tomotherapy,” Semin. Radiat. Oncol. 9, 108-117 (1999).

${ }^{13}$ J. S. Aldridge, "Tomographic patient registration and conformal avoidance tomotherapy," Ph.D. thesis Department of Medical Physics, University of Wisconsin-Madison, 1999.

${ }^{14}$ E. E. Fitchard et al., "Registration using tomographic projection files," Phys. Med. Biol. 44, 495-507 (1999).

${ }^{15}$ E. E. Fitchard, G. H. Olivera, K. J. Ruchala, J. M. Kapatoes, R. Hinderer, P. J. Reckwerdt, and T. R. Mackie, "Experimental study of six-parameter patient registration from helical fan beam data," Proceedings of the XIII International Conference on Computers in Radiotherapy, Heidelberg, Germany, edited by W. Schlegel and T. Bortfeld (Springer, Berlin, 2000), pp. 587-589.

${ }^{16}$ G. H. Olivera, E. E. Fitchard, P. J. Reckwerdt, K. J. Ruchala, and T. R. Mackie, "Delivery modification as an alternative to patient repositioning in tomotherapy," in Ref. 15, pp. 297-299.

${ }^{17}$ K. J. Ruchala, G. H. Olivera, J. M. Kapatoes, E. A. Schloesser, P. J. Reckwerdt, and T. R. Mackie, "Megavoltage CT image reconstruction of treatment data for tomotherapy verification," Phys. Med. Biol. 45, 35453562 (2000).

${ }^{18}$ K. J. Ruchala, G. H. Olivera, J. M. Kapatoes, E. A. Schloesser, P. J. Reckwerdt, and T. R. Mackie, "Megavoltage CT Imaging as a byproduct of multi-leaf collimator leakage," Phys. Med. Biol. 45, N61N70 (2000)

${ }^{19}$ K. J. Ruchala, G. H. Olivera, E. A. Schloesser, and T. R. Mackie, "Megavoltage CT on a tomotherapy system," Phys. Med. Biol. 44, 2597-2621 (1999).

${ }^{20}$ K. J. Ruchala, G. H. Olivera, J. M. Kapatoes, J. B. Smilowitz, E. A. Schloesser, D. W. Pearson, J. P. Balog, P. J. Reckwerdt, and T. R. Mackie, "Tomographic verification of tomotherapy-before, during and after treatment," in Ref. 15, pp. 314-316.

${ }^{21} \mathrm{~S}$. Webb, The Physics of Conformal Radiotherapy: Advances in Technology (IOP, Philadelphia, 1997).

${ }^{22}$ R. Bogaerts, A. Van Esch, R. Reymen, and D. Huyskens, "A method to estimate the transit dose on the beam axis for verification of dose delivery with portal images," Radiother. Oncol. 54, 29-46 (2000).

${ }^{23}$ C. Fiorino, C. Uleri, G. M. Cattaneo, and R. Calandrino, "On-line exit dose profile measurement by a diode linear array," Phys. Med. Biol. 41, 1291-1304 (1996).

${ }^{24}$ J. Van Dam, C. Vaerman, N. Blanckaert, G. Leunens, A. Dutreix, and E. van der Schueren, "Are port films reliable for in vivo exit dose measurements?," Radiother. Oncol. 25, 67-72 (1992).

${ }^{25}$ J. W. Wong, E. D. Slessinger, R. E. Hermes, C. J. Offutt, T. Roy, and M. W. Vannier, "Portal dose images. I. Quantitative treatment plan verification," Int. J. Radiat. Oncol., Biol., Phys. 18, 1455-1463 (1990).

${ }^{26}$ X. Ying, L. Y. Geer, and J. W. Wong, “Portal dose images. II. Patient dose estimation,” Int. J. Radiat. Oncol., Biol., Phys. 18, 1465-1475 (1990).

${ }^{27}$ D. Huyskens, J. Van Dam, and A. Dutreix, "Midplane dose determination using in vivo dose measurements in combination with portal imaging," Phys. Med. Biol. 39, 1089-1101 (1994).

${ }^{28}$ J. H. Lanson, M. Essers, G. J. Meijer, A. W. H. Minken, G. J. Uitewaal, and B. J. Mijnheer, "In vivo dosimetry during conformal radiotherapy: Requirements for and findings of a routine procedure,' Radiother. Oncol. 52, 51-59 (1999).

${ }^{29}$ G. Leunens, J. Van Dam, A. Dutreix, and E. van der Schueren, “Quality assurance in radiotherapy by in vivo dosimetry. 1. Entrance dose measurements, a reliable procedure," Radiother. Oncol. 17, 141-151 (1990)

${ }^{30}$ P. Mangili, C. Fiorino, A. Rosso, G. M. Cattanea, R. Parisi, E. Villa, and R. Calandrino, "In-vivo dosimetry by diode semiconductors in combination with portal films during TBI: Reporting a 5-year clinical experience," Radiother. Oncol. 52, 269-276 (1999).

${ }^{31}$ Y. Aoki, A. Akanuma, P. M. Evans, D. G. Lewis, E. J. Morton, and W.
Swindell, "A dose distribution evaluation utilizing megavoltage CT imaging system," Radiat. Med. 8, 107-110 (1990).

${ }^{32}$ V. N. Hansen, P. M. Evans, and W. Swindell, "The application of transit dosimetry to precision radiotherapy," Med. Phys. 23, 713-721 (1996).

${ }^{33}$ B. J. M. Heijman, K. L. Pasma, M. Kroonwijk, V. G. M. Althof, J. C. J. de Boer, A. G. Visser, and H. Huizenga, "Portal dose measurement in radiotherapy using an electronic portal imaging device (EPID)," Phys. Med. Biol. 40, 1943-1955 (1995).

${ }^{34}$ M. C. Kirby and P. C. Williams, "The use of an electronic portal imaging device for exit dosimetry and quality control measurements," Int. J. Radiat. Oncol., Biol., Phys. 31, 593-603 (1995).

${ }^{35}$ M. Kroonwijk, K. L. Pasma, S. Quint, P. C. M. Koper, A. G. Visser, and B. J. M. Heijman, "In vivo dosimetry for prostate cancer patients using an electronic portal imaging device (EPID); demonstration of internal organ motion," Radiother. Oncol. 49, 125-132 (1998).

${ }^{36}$ K. L. Pasma, M. Kroonwijk, S. Quint, A. G. Visser, and B. J. M. Heijmen, "Transit dosimetry with an electronic portal imaging device (EPID) for 115 prostate cancer patients," Int. J. Radiat. Oncol., Biol., Phys. 45, 1297-1303 (1999).

${ }^{37}$ R. Boellaard, M. Van Herk, H. Uiterwaal, and B. Mijnheer, "First clinical test using a liquid filled electronic portal imaging device and a convolution model for verification of the midplane dose,"' Radiother. Oncol. 47, 303-312 (1998).

${ }^{38}$ M. Essers, M. Boellaard, M. van Herk, and B. J. Mijnheer, "Transmission dosimetry with a liquid-filled electronic portal imaging device," Int. J. Radiat. Oncol., Biol., Phys. 34, 931-941 (1996).

${ }^{39}$ H. Keller, M. Fix, and P. Ruegsegger, "Calibration of a portal imaging device for high-precision dosimetry: A Monte Carlo study," Med. Phys. 25, 1891-1909 (1998).

${ }^{40}$ T. R. McNutt, T. R. Mackie, P. J. Reckwerdt, N. Papanikolaou, and B. R. Paliwal, "Calculation of portal dose using the convolution/superposition method," Med. Phys. 23, 527-535 (1996).

${ }^{41}$ F. Yin, M. C. Schell, and P. Rubin, "Input/output characteristics of a matrix ion-chamber electronic portal imaging device," Med. Phys. 21, 1447-1454 (1994).

${ }^{42}$ Y. Zhu, X.-G. Jiang, and J. Van Dyk, "Portal dosimetry using a liquid ion chamber matrix: Dose response studies," Med. Phys. 22, 1101-1106 (1995).

${ }^{43}$ D. A. Jaffray, D. G. Drake, M. Moreau, A. A. Martinez, and J. W. Wong, "A radiographic and tomographic imaging system integrated into a medical linear accelerator for localization of bone and soft-tissue targets," Int. J. Radiat. Oncol., Biol., Phys. 45, 773-789 (1999).

${ }^{44}$ J. M. Kapatoes, G. H. Olivera, K. J. Ruchala, P. J. Reckwerdt, J. B. Smilowitz, and T. R. Mackie, "The limitations of dose reconstruction without treatment imaging," Proceedings of the World Congress on Medical Physics and Biomedical Engineering, Chicago, IL (unpublished).

${ }^{45}$ J. M. Kapatoes, G. H. Olivera, K. J. Ruchala, H. Keller, P. J. Reckwerdt, and T. R. Mackie, "Knowledge of the 3D treatment-position anatomy: A prerequisite for accurate dose reconstruction," Int. J. Radiat. Oncol., Biol., Phys. (submitted).

${ }^{46}$ J. M. Kapatoes, G. H. Olivera, K. J. Ruchala, P. J. Reckwerdt, J. B. Smilowitz, J. P. Balog, D. W. Pearson, and T. R. Mackie, "Database energy fluence verification and the importance of on-board CT imaging in dose reconstruction" in Ref. 15, pp. 294-296.

${ }^{47}$ H. H. Barrett and W. Swindell, Radiological Imaging (Academic, New York, 1981).

${ }^{48}$ K. L. Pasma, B. J. M. Heijman, M. Kroonwijk, and A. G. Visser, "Portal dose image (PDI) prediction for dosimetric treatment verification in radiotherapy. I. An algorithm for open beams,"’ Med. Phys. 25, 830-840 (1998).

${ }^{49}$ W. Swindell and P. M. Evans, "Scattered radiation in portal images: A Monte Carlo simulation and simple physical model," Med. Phys. 23, 63-73 (1996)

${ }^{50}$ V. N. Hansen, W. Swindell, and P. M. Evans, "Extraction of primary signal from EPIDs using only forward convolution," Med. Phys. 24, 1477-1484 (1997)

${ }^{51}$ R. L. Siddon, "Calculation of the radiological depth,' Med. Phys. 12, 84-87 (1985)

${ }^{52}$ R. L. Siddon, "Prism representation: A 3D ray-tracing algorithm for radiotherapy,"' Phys. Med. Biol. 30, 817-824 (1985).

${ }^{53}$ R. L. Siddon, "Fast calculation of the exact radiological path for a three- 
dimensional CT array,"' Med. Phys. 12, 252-255 (1985).

${ }^{54}$ J. P. Balog, T. R. Mackie, P. Reckwerdt, M. Glass, and L. Angelos, "Characterization of the output for helical delivery of intensity modulated slit beams," Med. Phys. 26, 55-64 (1999).

${ }^{55} \mathrm{~J}$. Balog, "Tomotherapy dosimetry and the tomotherapy benchtop," Ph.D. thesis, Department of Medical Physics, University of WisconsinMadison, 1998.

${ }^{56}$ G. H. Olivera, D. Shepard, P. J. Reckwerdt, K. J. Ruchala, J. Zachman, E. E. Fitchard, and T. R. Mackie, "Maximum likelihood as a common com- putational framework in tomotherapy,' Phys. Med. Biol. 43, 3277-3294 (1998).

${ }^{57}$ J. N. Yang, T. R. Mackie, P. Reckwerdt, J. O. Deasy, and B. R. Thomadsen, "An investigation of tomotherapy beam delivery," Med. Phys. 24, 425-436 (1997).

${ }^{58}$ M. Carol, W. H. Grant, A. R. Bleier, A. A. Kania, H. Targovnik, E. B. Butler, and S. W. Woo, "The field-matching problem as it applies to the peacock three dimensional conformal system for intensity modulation," Int. J. Radiat. Oncol., Biol., Phys. 34, 183-187 (1996). 